\title{
Linx
}

Revue des linguistes de l'université Paris X Nanterre

9 | 1997

Émile Benveniste. Vingt ans après

\section{La croisée des chemins. Remarques sur la topologie des relations langue / discours chez Benveniste}

Sarah de Vogüé

\section{OpenEdition}

Journals

Édition électronique

URL : http://journals.openedition.org/linx/1027

DOI : 10.4000/linx.1027

ISSN : 2118-9692

Éditeur

Presses universitaires de Paris Nanterre

Édition imprimée

Date de publication : 1 avril 1997

Pagination : 145-158

ISSN : 0246-8743

\section{Référence électronique}

Sarah de Vogüé, «La croisée des chemins. Remarques sur la topologie des relations langue / discours chez Benveniste », Linx [En ligne], 9 | 1997, mis en ligne le 25 juillet 2012, consulté le 01 mai 2019. URL : http://journals.openedition.org/linx/1027 ; DOI : 10.4000/linx.1027 


\title{
La croisée des chemins Remarques sur la topologie des relations langue / discours chez Benveniste
}

\author{
Sarah de Vogüé
}

"[...] bien que leurs chemins se croisent à tout moment." (I, 130, Les niveaux de l'analyse linguistique)

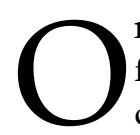

n est avec la théorie de Benveniste face à un paradoxe que l'on peut formuler de la façon suivante : la dichotomie sémiotique / sémantique qui traverse la langue et qui à ce titre est interne à la langue, séparant deux manières d'être langue ("la langue comme sémiotique", "la langue comme sémantique" II, 225, La forme et le sens dans le langage), intègre cependant, à travers son versant sémantique, un hors la langue coextensif à son emploi dans le discours. Ainsi, quand le sémiotique renvoie à la "réalité intrinsèque de la langue", le sémantique se trouve "relié" aux choses hors la langue". Quand le sémiotique se définit comme une "propriété de la langue", le sémantique "résulte d'une activité du locuteur qui met en action la langue" (ibidem). D'où deux univers distincts: le monde clos des signes d'un côté et "le monde de l'énonciation et l'univers du discours" (II, 64, Sémiologie de la langue) de l'autre. Le paradoxe est alors que ces deux univers recouvrent néanmoins une même réalité : la langue. Dès lors le discours est en même temps opposé à la langue comme clôture, et inscrit dans la langue.

Ce paradoxe, qui parcourt l'ensemble des Problèmes de linguistique générale, et qui paraît constituer le nœud autour duquel la pensée de Benveniste s'organise, se manifeste dans le texte même des articles rassemblés, à travers ce qui prend la forme d'une figure de style, où une thèse étant posée, elle se trouve en quelque sorte retournée, pour intégrer ce qui serait son envers. Je citerai pour exemple la formule célèbre de la subjectivité dans le langage: "L'installation de la subjectivité dans le langage crée dans le langage et, croyons-nous, hors du langage aussi bien, la catégorie de la personne." (I, 263). Elle me paraît en effet exprimer sous sa forme la plus condensée cette 
Sarah de Vogüé

figure singulière où dans un battement - ici, une simple respiration, que l'insertion du modalisateur croyons-nous vient imprimer à la phrase - la pensée revient sur elle-même pour englober son extérieur ${ }^{1}$.

Ce battement entre le dans la langue et le hors la langue a pu être donné comme le produit d'une pensée confuse ("brouillée" dit Culioli dans Emile Benveniste aujourd'hui) ou contradictoire (c'est le point de vue d'Irène TambaMecz dans le même recueil). Il a pu être rapporté, de façon moins critique, à la position intenable qui serait celle de Benveniste, entre continuité et dépassement de Saussure (Claudine Normand dans son article de 89 parle de la difficulté intrinsèque du projet).

Je crois pour ma part qu'on peut être à la fois à l'intérieur et à l'extérieur. Et je me fonde, pour le croire, sur les lois de la topologie qui, en énonçant les propriétés générales des lieux, posent la possibilité d'un espace qui soit à la fois intérieur et extérieur. J'en prendrai pour exemple cet objet pour le moins paradoxal (comme le sont bien d'autres objets topologiques), et en un sens inconcevable, qu'est la bouteille de Klein. Un objet que l'on peut représenter comme une bouteille qui entrerait dans elle-même, de manière que, passant toujours continûment de l'intérieur à l'extérieur, on soit en tout point à la fois à l'intérieur et à l'extérieur de la bouteille.

Ainsi n'est-il pas exclu que le paradoxe de l'intériorité / extériorité du discours par rapport à la langue soit dans la nature même de la langue (et non dans l'esprit brouillé de Benveniste). Il y aurait cet impensable dans la langue, et c'est cet impensable que Benveniste se serait fixé la tâche de penser. C'est en tous les cas ce que lui reconnaît Ricœur dans le débat qui suit La forme et le sens dans le langage (voir l'hommage que ce dernier lui rend p. 236).

\section{L'intégration}

J'ai essayé dans un précédent article (de Vogüé 1993) de caractériser ce rapport spécifique entre intérieur et extérieur à partir du concept d'intégration

\footnotetext{
${ }^{1}$ Mais la figure du battement prend d'autres formes quand, dans un même texte, ou d'un texte sur l'autre, des formules se répondent, pour s'inverser. Le cas le plus emblématique est celui des deux citations qui viennent clore respectivement Les niveaux de l'analyse linguistique et La forme et le sens dans le langage, l'une en latin, l'autre en grec, l'une "calquant une formule classique", l'autre d'Héraclite, "limpide et mystérieuse", à propos du Seigneur de l'oracle de Delphes :

"nihil est in lingua quod non prius fuerit in oratione." (I, 131)

"Oute légei, oute kryptei 'Il ne dit, ni ne cache', alla semainei 'mais il signifie'." (II, 229)

La seconde se trouvant étayée par la phrase magistrale qui conclut le paragraphe précédent :

"Mais au fondement de tout, il y a le pouvoir signifiant de la langue, qui passe bien avant celui de dire quelque chose."
} 
qui en donnerait la formule. Le terme d'intégration somme toute assez peu employé par Benveniste, intervient essentiellement à deux endroits :

- d'une part pour caractériser le rapport du mot (ou du signe, j'y reviendrai) à la phrase (ou au syntagme) :

"Le sens d'un mot consistera dans sa capacité d'être l'intégrant d'un syntagme." (II, 227, La forme et le sens dans le langage)

- d'autre part pour caractériser le rapport entre langue et culture :

"un rapport d'intégration nécessaire." (II, 24, Structuralisme et linguistique)

Dans ce second contexte, on peut le tenir pour partiellement équivalent aux termes d'englobement, d'inclusion, et aussi, dans sa forme verbale intégrer, au verbe contenir, qui reviennent quant à eux massivement pour caractériser le rapport entre langue et culture, langue et société, langue et autres systèmes sémiologiques, entre la langue et le monde humain de manière générale.

On peut donc considérer (c'est en tous les cas la démonstration de Claudine Normand dans son article de 89) qu'il renvoie à la formule plus explicite définissant le rapport entre langue et société dans Structure de la langue et structure de la société :

"Premièrement, la langue est l'interprétant de la société. Deuxièmement, la langue contient la société." (II, 95)

Cette formule (où la conjonction de deux propositions toutes deux données comme essentielles, mais qui d'une certaine façon s'opposent, relève à nouveau de ce que j'ai appelé ci-dessus la figure du battement) peut à son tour être rapprochée du passage suivant de Sémiologie de la langue :

«Si l'on s'interroge sur la situation respective de la langue et de la société (...), le sociologue [...] observera que la langue fonctionne à l'intérieur de la société qui l'englobe [...]. Mais la considération sémiologique inverse ce rapport, car seule la langue permet la société. La langue constitue ce qui tient ensemble les hommes, le fondement de tous les rapports qui à leur tour fondent la société. On pourra dire alors que c'est la langue qui contient la société. Ainsi la relation d'interprétance, qui est sémiologique, va à l'inverse de la relation d'emboîtement, qui est sociologique." (II, 62)

J'ai proposé d'analyser cette relation d'intégration en prenant en compte l'ambivalence propre au terme même d'intégration qui, dans son usage courant, désigne aussi bien un procès d'englobement (intégrer un individu) qu'un procès d'insertion (intégrer un ensemble). C'est d'ailleurs plutôt le procès 
d'englobement qu'il désigne dans le second emploi, et le procès d'insertion qu'il désigne dans le premier.

Dans les deux emplois cependant, on passe, par une forme de retournement, de l'un des sens du terme à l'autre. Dans le premier, cette capacité d'insertion, en tant qu'elle définit le sens des unités, introduit au fondement même de l'unité l'ensemble de ses contextes d'insertion : l'insertion est aussi englobement. Dans le second, l'englobement n'est qu'interprétance, et n'est que le retournement de l'inclusion sociologique.

Il peut paraître étrange de faire tenir ensemble les réflexions de Benveniste sur le rapport langue / société et celles sur le rapport mot (ou signe) / syntagme. C'est que, pour Benveniste, il s'agit dans les deux cas d'un rapport entre la langue et son extérieur même si l'extérieur est différent.

J'en déduis que le terme d'intégration désigne très précisément ce rapport paradoxal entre intérieur et extérieur que j'essaye d'élucider ici. Par son ambivalence, il procède de cette même figure du battement que je repère dans différentes formules des PLG. Simplement le battement se trouve là condensé en un seul mot.

J'ai essayé dans l'article de 92 de donner la formule de ce battement de l'intégration, pour ce qui concerne le rapport langue / société (ou plus généralement langue / monde humain) :

"L'intérieur langue contient son extérieur dans la mesure où il l'interprète, dans son extériorité maintenue mais transformée ou intégrée."

Dans la relation signe / syntagme, on peut penser que c'est le signe (l'intégré) qui se trouve transformé, et qui devient ainsi mot. Ce serait du moins une façon d'articuler ensemble le signe et le mot tels qu'ils se voient différenciés dans La forme et le sens dans le langage ${ }^{2}$.

On vient de voir jouer le battement de l'intégration en deux endroits : entre la langue comme monde clos des signes (sémiotique) et la phrase (prise dans le sémantique) d'une part, entre la langue prise globalement (langue et discours, sémiotique et sémantique) et son extérieur (société, culture, monde humain) d'autre part. C'est là le début de la chaîne et le bout de la chaîne de ce grand processus d'englobement / retournement qui va du signe / mot au monde humain.

${ }^{2}$ Rappelons que dans Les niveaux de l'analyse linguistique, Benveniste les tient pour équivalents. 
Au centre du processus, il y a le sémantique, c'est-à-dire non seulement les phrases, mais l'ensemble de la dimension énonciative ${ }^{3}$. Que le sémantique soit globalement intégré dans la langue, par delà son extériorité, c'est ce qu'indique la formule "la langue comme sémantique" dont nous sommes partis. J'expliciterai ci-dessous quelques-uns des éléments qui me paraissent fonder cette intégration, dont il faudra aussi mesurer les conséquences sur l'interprétation à donner au concept même de sémantique.

\section{Le sémantique dans la langue}

2.1. Il est un point sur lequel Benveniste n'a jamais faibli : la phrase relève de l'univers sémantique. Il est frappant d'observer comment les intervenants dans le débat qui suit La forme et le sens dans le langage, et Ricœur lui-même, s'acharnent à contester cette appartenance de la phrase au sémantique. Sous couvert de reprocher à Benveniste une conception trop étriquée du discours (Perelman), une conception trop simple de la phrase (Gochet) ou une conception trop limitée de la langue (Ricœur), leur propos commun semble être de réancrer la phrase (ou du moins la part grammaticale de la phrase, ce qui la distinguerait de l'énoncé) au sein du système de la langue (qui serait alors un sémiotique élargi puisqu'il ne se résumerait pas à un système de signes). Peut alors se déployer ce qui n'est plus qu'une simple pragmatique, à savoir le sémantique dont parle Benveniste, mais dégonflé, expurgé de ce qui en fait la substance, réduit à la seule dimension énonciative : l'utilisation de la langue par un locuteur. Le bénéfice de l'opération est immédiat: ce sémantique sans les phrases peut alors être définitivement séparé de la langue, totalement extérieur.

Il est clair en effet que dès que le sémantique comprend les phrases, toute phrase, le processus de fabrication des phrases, il est inclus dans la langue. Et le paradoxe dont nous sommes partis s'ensuit: le sémantique est inclus dans la langue, bien qu'il soit relié à son extérieur par ces points d'extériorité que sont le locuteur et le référent, et qu'est globalement l'énonciation.

Il est intégré à la langue, parce qu'il n'est pas la pragmatique.

2.2. Mais il y a une autre raison pour laquelle le sémantique de Benveniste n'est pas la pragmatique. Une raison, pourrait-on dire, en aval du problème des phrases, qui concerne l'énonciation elle-même. L'extériorité de l'énonciation par rapport à la langue est remise en cause à son tour, et elle l'est par le mécanisme même de l'énonciation, tel que Benveniste le décrit.

${ }^{3}$ Chez Benveniste, les deux vont de pair : il n'y a de phrase hors d'une prédication, et il n'y a de prédication hors de la dimension énonciative. 
Sarah de Vogüé

Le locuteur qui s'approprie la langue pour produire un discours est bien a priori extérieur à la langue. Pourtant, il s'avère qu'il est lui aussi dans la langue et hors la langue.

Le retournement s'opère là en trois temps :

1) Le locuteur s'approprie la langue dans l'acte d'énonciation.

2) Il est alors "introduit dans sa parole" (II, 82, L'appareil formel de l'énonciation).

3) Ainsi est-il par conséquent "créé" comme sujet, à travers l'indicateur je, "dans le langage et hors du langage aussi bien" (I,262-263, De la subjectivité dans le langage)

Que le sujet soit créé par le discours "hors du langage aussi bien", c'est là une thèse extrêmement forte. On connaît l'argumentation qu'en donne Benveniste :

"C'est dans l'instance de discours où je désigne le locuteur que celui-ci s'énonce comme "sujet". [...] Si l'on veut bien y réfléchir, on verra qu'il n'y a pas d'autre témoignage objectif de l'identité du sujet que celui qu'il donne ainsi sur lui-même. " (I, 262, De la subjectivité dans le langage)

Mais cela vaut aussi du présent de l'énonciation, rapporté au "temps linguistique", parce qu'il est créé par la langue, ce qui le distingue de facto du moment "physique" où l'énoncé est proféré : il est "réinventé chaque fois qu'un homme parle." (II, 74, Le langage et l'expérience humaine)

Ainsi est-ce l'ensemble des paramètres de l'énonciation qui, par delà leur extériorité, sont pris dans la langue d'être créés par le discours.

\section{Le sémantique dans le sémiotique}

3.1. Il faut aller plus loin dans l'intrication entre langue et discours. On a vu en 1 que le concept d'intégration intervenait dès Les niveaux de l'analyse linguistique, pour rendre compte du rapport entre signes et syntagmes. C'est dire qu'au moment même où Benveniste pose la séparation des deux univers que sont le sémiotique, dont l'unité est le signe, et le sémantique, dont l'unité est la phrase, le battement de l'intégration vient subvertir cette séparation. Les signes, de par leur fonction intégrative, intègrent les phrases. Non seulement le sémantique est intérieur à la langue prise globalement, mais il est intégré à la langue comme système - au sémiotique. Et ce nouage entre les deux univers est crucial dans l'article, puisque le signe est alors défini en tant qu'unité sémiotique (distinctive) par cette fonction intégrative qui est ce qui constitue 
son sens ${ }^{4}$. Le sémiotique est donc fondé sur l'intégration du sémantique. D'où la citation latine qui conclut l'article (voir note 1), où le discours est donné comme premier.

Il faut noter que La forme et le sens dans le langage constitue sur ce point un revirement. En renonçant à définir le signe par sa fonction intégrative, en excluant les relations syntagmatiques du champ du sémiotique, en différenciant les signes (sémiotiques) des mots (sémantiques), ce texte vise d'une certaine façon à consommer la séparation des deux univers, bien que ces signes et ces mots soient les mêmes entités, Benveniste y insiste, pris dans des univers différents.

La citation d'Héraclite qui clôt la conférence (et qui répond à la citation latine des Niveaux, voir note 1) éclaire les raisons de ce revirement. Derrière ce qui me paraît constituer un recul par rapport au premier texte, il y a la crainte de voir le sémiotique se dissoudre dans cette inclusion du sémantique et d'un discours qui le fonderait. "Au départ de tout, il y a le pouvoir signifiant de la langue, qui passe bien avant celui de dire quelque chose." (II, 229) Les thèses défendues dans Les niveaux ne peuvent être soutenues qu'à condition de ne pas réduire l'intégration à un englobement: l'extériorité du sémantique doit être maintenue. Si le discours fonde le signe, ce ne peut être qu'un discours virtuel, un champ de discours possibles, l'ensemble des discours que le signe intègre de par son sens, mais qui ne sauraient s'actualiser hors de l'appropriation de la langue par un locuteur.

3.2. L'intégration du sémantique dans le sémiotique joue à un autre niveau. Cela concerne cette fois, plus localement, les signes "vides" de l'appareil formel de l'énonciation: les marques de personne, les marques temporelles entre autres.

Ces signes vides, quoiqu'appartenant au système de la langue (au sémiotique), se définissent par leur fonction dans la structuration énonciative (sémantique). Ils ne signifient que par l'énonciation.

Il ne faut pas y voir une faiblesse du modèle. D'ailleurs, si c'était une faiblesse, Benveniste ne la revendiquerait pas aussi explicitement (voir en particulier I, 263, De la subjectivité dans le langage). Il n'a jamais été dit que sémiotique et sémantique avaient des objets différents: seules les méthodes diffèrent, et les principes régissant les deux ordres. Que les signes vides se définissent par leur fonction dans la structuration énonciative n'est qu'un retournement de plus du modèle.

\footnotetext{
4 Je retrace dans de Vogüé 1993 la démonstration que Benveniste donne de ce point crucial.
} 
Sarah de Vogüé

En fait, le retournement est ici complet (la boucle se boucle) parce que ces signes sont aussi ce qui rend possible l'énonciation. D'où la boucle en trois temps suivante, qui prolonge celle que l'on a retracée en 2.2. :

1) Le locuteur s'approprie la langue.

2) Il est ainsi introduit dans sa parole, instanciant les signes vides.

3) Ces signes vides, instaurant par conséquent les conditions de l'intersubjectivité, sont ce qui "permet" que le locuteur s'approprie la langue (I, 262, De la subjectivité dans le langage).

\section{Le battement de l'histoire}

4.1. A propos de l'histoire aussi il y a battement. A priori ce battement ne concerne pas le rapport sémiotique / sémantique. Il concerne seulement l'énonciation, non plus dans sa relation à la langue, mais dans sa relation au sujet. On peut le formuler ainsi: il est des énonciations - les énonciations historiques - qui ne relèvent pas de l'énonciation d'un sujet.

Cela est lié au double sens qui est conféré au terme de discours : défini en opposition à l'histoire dans Les relations de temps dans le verbe français, il renvoie ailleurs à l'acte même d'appropriation de la langue par un sujet, englobant alors discours et histoire. Ainsi l'histoire est et n'est pas du discours, est dans le discours et hors du discours.

On pourrait considérer que ces deux discours, celui qui englobe toute énonciation dont l'histoire, et celui qui s'oppose à l'histoire, ne sont pas les mêmes (de la même façon que l'on pourrait considérer que la langue comme système de signes et la langue englobant sémiotique et sémantique ne sont pas les mêmes). On pourrait n'y voir qu'une nouvelle confusion de la pensée de Benveniste, employant le même mot pour deux entités distinctes. Mais la récurrence du procédé (le même glissement qui opère de langue à langue opère ici de discours à discours) suffit à indiquer qu'il s'agit plus d'une figure de pensée que d'une simple maladresse. Surtout, les deux discours ne sont pas sans rapport (comme les deux langues n'étaient pas sans rapport), se définissant l'un et l'autre par l'énonciation : l'un est le produit de l'énonciation, acte par lequel le locuteur s'approprie la langue (le discours "au sens large"), l'autre réfère à l'énonciation, le locuteur se trouvant alors "introduit dans sa parole" (le discours opposé à l'histoire). Dans l'identification du premier au second, on retrouve par conséquent les temps 1 et 2 de la boucle explicitée en 3.2. Le glissement d'un discours à l'autre n'est que l'une des formes que prend 
le retournement par lequel l'énonciation est intégrée à l'énoncé (elle devient le référent de l'énoncé produit), avant que de l'être à la langue (temps 3) 5 .

Qu'il y ait cependant deux discours, signifie que l'énonciation est et n'est pas sui-référentielle. On a ainsi, d'un texte à l'autre, contradiction entre deux thèses :

1) "Une donnée constitutive de l'énonciation [...] est qu'elle introduit celui qui parle dans sa parole" (II, 82, L'appareil formel de l'énonciation).

ou

"Une phrase participe toujours de l'ici-maintenant" (II, 226, La forme et le sens dans le langage)

2) L'histoire met en scène "l'événement hors de la personne d'un narrateur" (I, 241, Les relations de temps dans le verbe français), et est définie par opposition au discours décrit quant à lui comme "toute énonciation supposant un locuteur et un auditeur (ibidem, 242), ce qui signifie que d'autres énonciations, précisément les énonciations historiques, ne les supposent pas ${ }^{6}$.

A nouveau, on pourrait mettre la contradiction au compte d'une insuffisance de Benveniste qui n'aurait pas su encore distinguer entre locuteur et énonciateur, ou, pour l'histoire, entre locuteur et narrateur (qui n'aurait pas lu Genette) : il y aurait toujours un repérage subjectif dans l'histoire, mais que Benveniste n'aurait pas perçu, car il s'organiserait autour du narrateur.

\footnotetext{
5 Un autre argument pour soutenir qu'il n'y a qu'un seul discours derrière les deux discours distingués (et une seule langue derrière les deux langues distinguables) serait que ces deux discours reçoivent précisément le même nom. L'argument est insuffisant. Il est un autre nom récurrent dans les textes de Benveniste qui désigne deux voire trois entités distinctes : c'est celui de sujet, qui réfère dans certains articles au locuteur, dans d'autres articles au sujet grammatical des phrases, parfois à un sujet psychologique, siège de pulsions émotionnelles diverses (c'est le cas dans Pour une sémantique de la préposition allemande vor). Je ne pense pas pour ma part que l'on puisse considérer ces trois entités comme identiques. Benveniste ne fait que suivre là un usage des plus traditionnels (alors que son usage du mot discours est le fruit d'une élaboration conceptuelle explicite), et rien dans les caractérisations qu'il peut donner de ces trois sujets n'indique qu'ils puissent être reliés. Ainsi je ne confondrais pas le voilier dont il est question dans Pour une sémantique de la préposition allemande vor (II, 140), et qui est sujet grammatical de la locution vor dem wind segeln, avec le sujet locuteur : ils n'ont tout simplement pas les mêmes propriétés.

6 Dans La nature des pronoms, le même battement concernant la sui-référentialité opère cette fois sur une seule page :

"On doit insister sur ce point : la 'forme verbale' est solidaire de l'instance individuelle de discours en ce qu'elle est toujours et nécessairement actualisée par l'acte de discours et en dépendance de cet acte. [...]

Mais en est-il toujours ainsi ? [...] Nous savons empiriquement que non. Il y a des énoncés de discours, qui en dépit de leur nature individuelle, échappent à la condition de personne, c'est-à-dire renvoient non à eux-mêmes mais à une situation 'objective'. C'est le domaine de ce que l'on appelle la 'troisième personne'." (I, 255)
} 
Sarah de Vogüé

Mais réintégrer un narrateur dans l'histoire, comme l'a fait Genette et comme l'ont fait bien d'autres à sa suite, revient à perdre ce qui fait la spécificité de l'histoire selon Benveniste. Une histoire où "Personne ne parle", où "il n'y a même plus alors de narrateur", où "les événement sont posés comme ils se sont produits à mesure qu'ils apparaissent à l'horizon de l'histoire", où "les événements semblent se raconter eux-mêmes".

D'ailleurs Benveniste oppose explicitement à différentes reprises histoire et narration. Ainsi il rejette le terme de temps narratif pour décrire le passé simple parce que le parfait peut être un temps narratif (note 2, I, 242). De même, dans Le langage et l'expérience humaine, il distingue les deux passés du français pour les rapporter l'un à l'histoire et l'autre à la narration (II, 74).

Il y a donc bien une énonciation qui, selon l'analyse de Benveniste, est dégagée du sujet et de la situation d'énonciation: une énonciation hors énonciation. Ou, pour le dire autrement, il y a une référence hors du discours, hors de l'allocution. L'énonciation n'est pas toujours sui-référentielle. Cela ne concerne d'ailleurs pas que l'histoire, mais aussi toute la sphère de la troisième personne (voir note 6). Dans Structure de la langue et structure de la société, Benveniste va même plus loin : c'est la troisième personne qui, en permettant une -référence hors allocution, est au fondement de l'opération même de référence :

"L'opposition moi-toi / lui [...] effectue l'opération de la référence et fonde la possibilité du discours sur quelque chose, sur le monde, sur ce qui n'est pas l'allocution." (II, 99)

4.2. Ce statut particulier de l'histoire, qui fait d'une certaine façon figure d'envers énonciatif, et transforme ce faisant de manière non négligeable le concept d'énonciation en introduisant la possibilité d'une énonciation sans origine, se trouve affecter aussi la relation établie entre sémiotique et sémantique. En effet, bien que la question de l'histoire ne soit traitée que dans le cadre de la réflexion sur cet univers de l'énonciation que Benveniste appellera plus tard sémantique, il me semble qu'elle n'est pas sans avoir des effets sur le sémiotique lui-même.

Ces effets, on les décèle dans La nature des pronoms, dans ce qui n'est peut-être cette fois qu'une réelle confusion conceptuelle, mais qui me paraît néanmoins mériter l'attention.

Tout l'article est fondé sur la distinction entre ce qui est décrit comme deux "modes de langage" (I, 251). C'est bien la dichotomie sémiotique / sémantique qui est là en jeu quoique les termes ne soient pas encore utilisés : "la langue comme répertoire de signes et système de leurs combinaisons, d'une 
part, et, de l'autre, la langue comme activité manifestée dans des instances de discours" (I, 257). La dichotomie permet d'opposer la troisième personne aux deux premières : elle structure donc le texte.

Or, ce clivage subit au cours de l'article des modifications. A la page 254, il est encore question de "deux plans", mais qui sont cette fois l'histoire et le discours: Benveniste parle de "temps et lieux 'historiques'" et oppose entre autres $i l$ et je, alors et maintenant, la veille et hier, caractéristiques respectivement de l'histoire et du discours. Par ailleurs, à la page 255, il est question de "la fonction dénominative du langage" d'un côté et "d'indicateurs autoréférentiels" de l'autre, le tout étant décrit comme le "double plan" sur lequel se constitue l'énoncé.

Il est surprenant de voir l'histoire ainsi mise du côté du sémiotique (sur le même "plan"). Et peut-être n'est-ce effectivement qu'une maladresse d'écriture, une forme de digression de Benveniste qui, parlant des deux plans du sémiotique et du sémantique, en viendrait, chemin faisant, à évoquer ce qui serait un autre clivage, entre deux autres plans, ceux de l'histoire et du discours.

Mais il est tout aussi surprenant de voir la fonction dénominative du langage, qui relève de la référence (cette fonction dénominative concerne explicitement les "références d'objet" (255)), mise du même côté que le système des signes. Car la référence est dès le départ rattachée à la dimension sémantique de la langue (voir dans La forme et le sens dans le langage: "[...] il faut introduire ici un terme qui n'était pas appelé par l'analyse sémiotique : celui de 'référent', indépendant du sens, et qui est l'objet particulier auquel le mot correspond dans le concret de la circonstance ou de l'usage." II, 226).

En fait, ce second point est surprenant sans l'être vraiment. Car le rattachement de la référence à la seule dimension sémantique ne se fait pas sans difficulté. Benveniste évoque à plusieurs reprises la référence en relation au mot: en relation à la "sémantique lexicale" dans La forme et le sens dans le langage p. 226, et en relation aux unités inférieures à la phrase, "se rapportant à des 'objets' généraux ou particuliers, pris dans l'expérience ou forgés par la convention linguistique" dans Les niveaux de l'analyse linguistique (I, 128). Et c'est seulement lorsqu'il distingue le signe du mot dans La forme et le sens dans le langage qu'il parvient à extraire du sémiotique toute problématique de la référence. Par ailleurs, on a vu en 4.1. que c'était précisément ce que permettait l'histoire et ce que permettait la troisième personne, que la langue ait une fonction dénominative hors de la situation d'énonciation. 
Sarah de Vogüé

L'histoire permet une référence hors l'énonciation, fondée simplement sur le système des signes que constitue le sémiotique ${ }^{7}$. L'histoire est donc un autre lieu de sémantisation du sémiotique, où le système des signes réfère tout seul, hors un sujet.

L'histoire est une autre croisée des chemins. Il s'agit cette fois d'une sémantisation en dehors de tout ancrage énonciatif, puisque, contrairement à ce qui est dit dans La forme et le sens dans le langage, où "le référent est toujours lié à une situation concrète, changeante (celle de l'énonciation)" (II, 227), la référence est cette fois hors situation. On comprend mieux alors que l'histoire puisse (par mégarde ?) apparaître sur le même "plan" que le sémiotique : ce n'est que dans l'histoire que le système de la langue peut signifier (et référer) par lui-même, hors un sujet se l'étant approprié.

"Personne ne parle ici. Les événements semblent se raconter euxmêmes". A cette formule qui n'est pas sans rappeler l'oracle de Delphes et la parole "limpide et mystérieuse du vieil Héraclite", je serais tentée d'ajouter "et les mots semblent parler tout seuls".

Ainsi, mais après combien de détours, via l'histoire comme envers de cet extérieur qu'est le discours, les signes réfèrent. Car peut-être est-ce dans l'histoire, dans ces textes sans sujet, que s'entend ce "pouvoir signifiant de la langue qui passe bien avant celui de dire quelque chose".

\section{Le programme sémantique}

Face à ces retournements incessants, on peut comprendre que le lecteur des PLG ressente quant à lui quelque confusion. Tout serait-il dans tout et réciproquement? Non, car au départ de tout il y a la langue. Mais une langue qui en chacun de ses points se retourne pour intégrer son extérieur.

Le projet de Benveniste de séparer deux linguistiques aux méthodologies distinctes apparaît-il ruiné par ces retournements? Non, bien que ces méthodologies distinctes n'aient pas fait l'objet d'une présentation synthétique de la part de Benveniste.

Certes, quand il travaille sur le sémiotique, sur les signes, il intègre, via leurs fonctions (dans le syntagme, dans le discours, dans la construction de la subjectivité) le sémantique. C'est l'effet des retournements.

Mais quand il travaille sur le sémantique, c'est un autre travail.

7 On comprend mieux ainsi la formulation étrange employée p. 255 de La nature des pronoms :

"Un énoncé personnel fini [...] met en œuvre la fonction dénominative du langage pour les références d'objet que celle-ci établit comme signes lexicaux distinctifs [...]". 
C'est un autre travail lorsqu'il travaille sur l'implicite (qui ne saurait être un signe). Le présent de l'énonciation est donné comme implicite ("le présent (...), signalé par la coïncidence de l'événement et du discours, est par nature implicite." II, 74, Le langage et l'expérience humaine). Le sujet lui-même peut être présent de manière implicite si l'on en croit la formule selon laquelle "introduire celui qui parle dans sa parole" est une "donnée constitutive de toute énonciation" (non historique) : il n'y a pas nécessairement je dans ces énonciations, et en particulier dans les phrases nominales que Benveniste rapporte explicitement au discours (La phrase nominale, I, 162-167). De même la prédication est donnée comme le bord implicite de la fonction verbale ( $L a$ phrase nominale, I, 155).

C'est aussi un autre travail quand il s'intéresse précisément à la prédication et à la fonction verbale (dans La phrase nominale, mais aussi dans Le système des relations de personne dans le verbe, où est déployée une théorie du sujet grammatical qui est assez singulière puisque le sujet y est décrit comme une forme d'"apposition" (I, 231)).

C'est clairement un tout autre travail quand il étudie un certain nombre de syntagmes: l'auxiliation, les composés, les délocutifs, les relatives, les dérivés en -eur et les syntagmes nominaux du type un bon danseur.

Le fait est qu'il s'en tient généralement à des syntagmes minimaux : en particulier ces composés et ces dérivés qui l'ont beaucoup occupé, ne sont presque plus des syntagmes puisqu'ils nourrissent le lexique.

Je pense qu'il faut y voir l'effet de cet attachement qui est le sien à étudier les relations entre sémiotique et sémantique. L'intégration dans le lexique de dérivés, la fabrication de composés, ne sont qu'une matérialisation particulièrement tangible des retournements analysés ici. Benveniste explique d'ailleurs dans Convergences typologiques (II, 104) qu'il s'intéresse au "rôle de la fonction prédicative dans la dérivation et la composition". Tel est bien le pari : que le prédicatif, les phrases, le discours (voir les délocutifs) soient présents, intégrés, dans le lexique. Face à ce pari, la dérivation et la composition, les transpositions qui s'y opèrent du discours en langue, constituent le laboratoire idéal.

De même que Benveniste ne peut étudier la dimension sémiotique de la langue sans croiser le sémantique, il ne peut étudier le sémantique sans retourner au sémiotique qui l'intègre. 
Sarah de Vogüé

\section{Bibliographie}

CULIOLI, Antoine, 1983, "Théorie du langage et théorie des langues", in Emile Benveniste aujourd'hui, Actes $d u$ colloque international $d u$ CNRS, Tour, Bibliothèque de L'information grammaticale.

NORMAND, Claudine, 1986, "Les termes de l'énonciation chez Benveniste", HEL, tome 8, fascicule II.

NORMAND Claudine, 1989, "Constitution de la sémiologie chez Benveniste", HEL, tome 11, Fascicule II.

TAMBA-MECZ, Irène, 1983, "A propos de la distinction entre sémiotique et sémantique", in Emile Benveniste aujourd'hui.

DE VOGÜÉ, Sarah, 1992, "Culioli après Benveniste", LINX, Nanterre. 